\title{
High-prevalence and broad spectrum of Cell Adhesion and Extracellular Matrix gene pathway mutations in epithelial ovarian cancer
}

\author{
Arash Rafii ${ }^{1,2 \dagger}$, Najeeb M Halabi ${ }^{1}$ and Joel A Malek ${ }^{2,3,4^{*}}$
}

\begin{abstract}
Background: Ovarian cancer is the most deadly gynecological cancer because of late diagnosis, frequently with diffuse peritoneal metastases. Recent findings have shown that serous epithelial ovarian cancer has a narrow mutational spectrum with TP53 being the most frequently targeted when single genes are considered. It is, however, important to understand which pathways as a whole may be targeted for mutation.
\end{abstract}

Findings: Previously published mutational data provided by the cancer genome atlas networks findings on ovarian cancer was searched for statistically significant enrichment of genes in pathways. These pathways were then searched in all patients to identify the spectrum of mutations. Statistical significance was further shown through in-silico permutations of exome sequences using empirically observed mutation rates. We detected mutations in the cell adhesion pathway genes in more than $89 \%$ of serous epithelial ovarian cancer patients. This level of near universal mutational targeting of the cell adhesion pathway, including the extracellular matrix pathway, is previously unreported in epithelial ovarian cancer.

Conclusions: Taken together with previous studies on the role of cell adhesion and extracellular matrix gene expression in ovarian cancer and metastasis, our results identify pathways for which the mutational prevalence has previously been overlooked using single gene approaches. Analysis of mutations at the pathway level will be critical in studying heterogeneous diseases such as ovarian cancer.

\section{Background}

Epithelial Ovarian Carcinoma (EOC) is the sixth most common malignancy in women [1] and has a poor overall survival rate (20 to $30 \%$ at 5 years). High-grade serous carcinoma is the most frequent type of ovarian cancer. The poor survival rate is due mainly to a large tumor burden and frequent, extensive peritoneal metastatic lesions at diagnosis. This results in difficulty to achieve complete optimal resection, despite advances in surgical practice. Indeed the importance of the metastatic process in ovarian cancer has been clearly demonstrated in the clinical setting by the fact that peritoneal residual disease at the end of the surgical treatment (even below $5 \mathrm{~mm}$ ) impacts prognosis [2,3].

\footnotetext{
*Correspondence: jom2042@qatar-med.cornell.edu

${ }^{\dagger}$ Equal contributors

2Department of Genetic Medicine, Weill Cornell Medical College, NY, NY, USA ${ }^{3}$ Genomics Core, Weill Cornell Medical College in Qatar, Education city, Qatar Foundation, Doha, Qatar

Full list of author information is available at the end of the article
}

Despite initial chemosensitivity and ultra-radical debulking surgery most patients will present with diffuse peritoneal recurrences rather than distant metastasis. Therefore, understanding the molecular mechanisms of progression from primary to metastases is critical for the development of effective therapies. Most studies of EOC have focused on the primary tumor including mutational and gene expression analysis. Many transcriptomic studies have been performed demonstrating different gene expression signatures depending on the histologic subtypes, the grade and stage [4-6]. Others have defined several prognosis signatures however there is only little overlap between these studies [7-9].

Recent results from the TCGA group's exome sequencing of 316 epithelial ovarian cancer primary tumors with matched controls revealed a very narrow spectrum for somatic mutations in EOC. Specifically, TP53 was mutated in approximately $96 \%$ of EOC primary tumors. The next most frequently affected genes were mutated

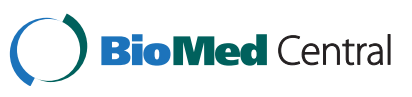


in less than $6 \%$ of tumors. This dramatic prevalence of TP53 mutations suggests an early and central role of TP53 mutations in EOC.

In our recent studies of copy number variations and gene expression differences between primary and metastatic lesions $[10,11]$ we observed clear targeting of pathways, rather than specific genes in EOC. Indeed our findings showed that analysis of pathways reduced the overall heterogeneity in comparisons and this pathwaybased approach may also be important in studying the mutational spectrum of ovarian cancer as well. The importance of pathway-based analysis over single gene analysis is due to the fact that similar downstream effects can be obtained by mutation of different genes within the same pathway. The TCGA study included some discovery-based pathway analysis on copy number and gene expression data using the PARADIGM approach. Analysis of mutations, however, was mainly restricted to certain known cancer pathways. The HOTNET approach overlaid the mutational data on protein interaction networks but no further discovery-based pathway analyses of gene mutations were reported [12].

We therefore hypothesized that analyzing the recent mutational data in EOC [12] using a broader functional pathway approach such as the Gene Ontologies or KEGG could reveal consistent targeting of pathways other than known cancer pathways, and could reduce the observed heterogeneity when only individual genes are considered. Furthermore, analysis of low frequency mutated genes within frequently mutated pathways, may offer insight into the metastatic process that only a few clones within the primary tumor undertake, but for which many patients are susceptible to.

\section{Methods}

Data was obtained from the TCGA study's supplementary information where a total of 316 EOC tumors were subjected to exome sequencing and the predicted mutations reported [12]. We selected genes that contained predicted non-silent mutations in at least 3 patients (mutated in at least $\sim 1 \%$ of patients). These genes were searched for enrichment in KEGG pathways and Gene Ontologies using DAVID [13] and GeneTrail [14]. These software packages offer robust statistical testing with appropriate multiple-hypothesis testing correction.

In-silico random mutagenesis was conducted using a sequence library comprising exons that overlap with the probes used in Agilent's All Exon $50 \mathrm{MB}$ kit. Custom scripts were written to introduce random mutations into these sequences and check for in-silico mutation rates in specific pathways. We used the mutation rates observed by the TCGA study: an AT mutation rate of $8.54 \times 10^{-7}$, $1.2 \times 10^{-6}$ for all GC bases, and $4.31 \times 10^{-6}$ for $\mathrm{CpG}$ repeats. Since insertion/deletions are not sequence specific, we added the rate of insertions/deletions $2.2 \times 10^{-7}$ to the other mutation categories. 100 permutations were conducted where each permutation consists of 316 random mutation runs mimicking the number of tumors analyzed by the TCGA. Data was gathered from the permutations to offer an empirically observed statistic.

To determine a pathways mutational spectrum and prevalence, each tumor's data was queried for potentially deleterious mutations in any genes containing a Gene Ontology annotation of 'Cell Adhesion' in the 'Molecular Function' category. All occurrences were recorded and summed across the pathway, for mutational spectrum, and set of patients for pathway mutation prevalence.

\section{Results}

Genes predicted to be mutated in at least 3 patients resulted in a list of 1382 genes that were most significantly enriched for Gene Ontology category "Cell Adhesion" (Benjamini-Hochberg adjusted p-value $=1.03 \times 10^{-25}$ ) with 156 genes identified. This was $\sim 2.5$ times more than 60 genes expected by random permutation. In KEGG, top enriched pathways included 'Extracellular Matrix (ECM)receptor interaction' (BH p-value $=3.35 \times 10^{-11}$ ) and 'Focal Adhesion' $\left(\mathrm{BH}\right.$ p-value $\left.=2.62 \times 10^{-9}\right)$ with 30 genes $(4.5 \mathrm{x}$ the expected 6.5 genes) and 45 genes $(2.9 \mathrm{x}$ the expected 15.5 genes) respectively. Both extracellular matrix and focal adhesion pathways share many of the same genes. Other pathways including 'Calcium signaling' were significantly enriched though less so than those mentioned above.

To more accurately estimate the significance of the TCGA observed mutations within the cell adhesion genes, we carried out an in-silico random mutagenesis simulation. If the frequency of random simulated mutations in cell adhesion genes is significantly less than the frequency of observed mutations in cell adhesion genes, this could suggest that there is selection in the tumor for cell adhesion mutations.

We ran 100 replicates of a trial where each trial consists of 316 random mutation runs mimicking the number of tumors analyzed by the TCGA. It is important to point out that this approach assumes equal mutational opportunity for the entire $50 \mathrm{Mb}$ capture region while typically only a portion of the targeted region is captured. This may result in some bias of the model. Nevertheless, our results (see Figure 1a), show that the frequency of simulated cell adhesion gene mutations in all 100 trials is significantly less (empirical p-value less than 0.01) than the frequency of observed cell adhesion gene mutations. This suggests that there is indeed a higher mutation rate in cell adhesion genes in actual ovarian tumors. 

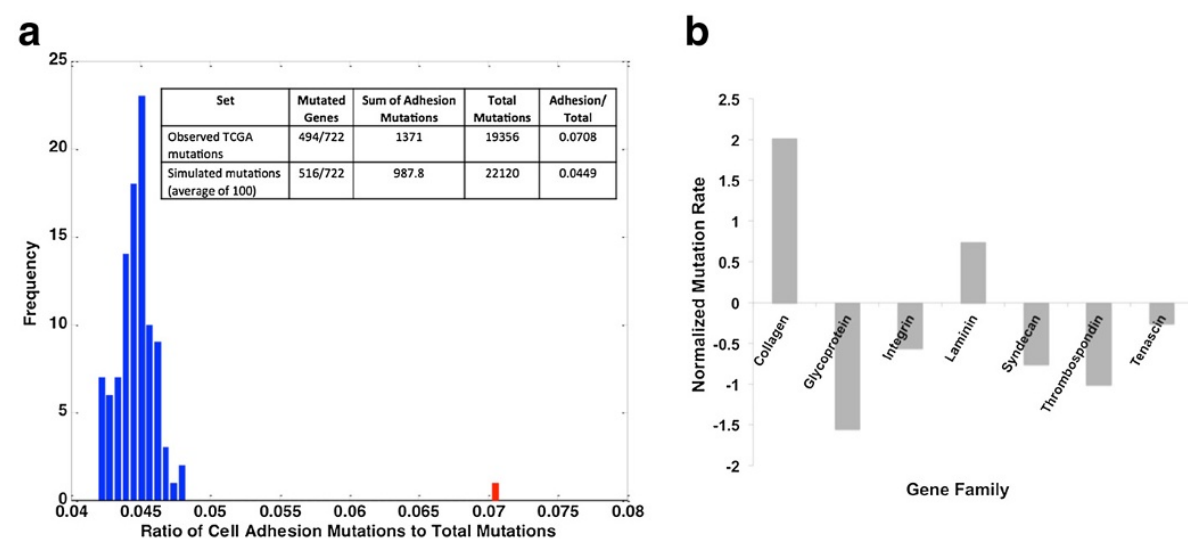

Figure 1 Simulated and observed Extracellular matrix gene family mutations in ovarian cancer. (a) Histogram showing the ratio of mutations in cell adhesion genes in the observed dataset (in red) and the ratio of mutations in cell adhesion genes to total mutations in simulated mutagenesis data (in blue). (b) Mutation rates were normalized to gene numbers in the family and compared to the baseline of all mutations in all genes. Collagens and Laminins had higher than expected mutation rates while the large gene family of Integrins had lower mutation rates.

Upon identifying cell adhesion genes as being highly enriched for mutations in the TCGA data, we determined the spectrum and the prevalence of mutations in this functional category among all patients. Each tumor's data was queried for all mutations, other than those predicted to be silent, in any of the 576 genes containing a Gene Ontology annotation of 'Cell Adhesion' in the 'Molecular Function' category. We found a broad spectrum of mutation in this category with 366 (64\%) of genes having a potentially deleterious mutation in at least one tumor (Table 1).

We further calculated the prevalence of 'Cell Adhesion' mutations among all patients' tumors. Strikingly, 281 of the $316(89 \%)$ tumors investigated had a mutation in at least one cell adhesion gene while 207 (66\%) tumors had mutations in at least two cell adhesion genes. This number of tumors with a mutation in the cell adhesion molecular function is comparable to the 283 automatically identified mutations in TP53. Results from other pathways are summarized in Table 1.
We also observed selection of certain gene families within functional categories that were targeted for mutation while other gene families were avoided. We identified all mutations in the KEGG pathway 'Extracellular Matrix (ECM)-receptor interaction' and normalized the number of mutations to the number of genes in selected gene families. A baseline ratio of all mutations to genes within the KEGG pathway was subtracted from each gene family (Figure 1b). We observed that collagens and laminins are especially targeted for mutation having $\sim 2$ and $\sim 1$ more mutations per gene than the baseline. Other categories including the integrins acquired fewer mutations than the average (Figure $1 \mathrm{~b}$ ).

\section{Conclusions}

The mutational spectrum in epithelial ovarian cancer is very narrow when only single genes are considered. TP53 mutations are near universal [12] with few other genes rising to significance. However, little work has been done to identify potential mutational targeting of

Table 1 Pathways enriched for non-synonymous SNPs in the TCGA data

\begin{tabular}{|c|c|c|c|c|c|c|c|}
\hline & $\begin{array}{l}\text { Molecular } \\
\text { Function/ } \\
\text { Pathway }\end{array}$ & $\begin{array}{l}\text { Enrichment } \\
\text { Rank by } \\
\text { p-value* }\end{array}$ & $\begin{array}{l}\text { Enrichment } \\
\text { P-value (BH } \\
\text { corrected) }\end{array}$ & $\begin{array}{l}\text { Enrichment } \\
\text { level (observed/ } \\
\text { expected) }\end{array}$ & $\begin{array}{l}\text { Pathway } \\
\text { mutational } \\
\text { spectrum:\% } \\
\text { of genes }\end{array}$ & $\begin{array}{c}\text { Pathway Mutational } \\
\text { Prevalence: } \% \\
\text { of tumors }\end{array}$ & $\begin{array}{l}\text { \# genes mutated } \\
\text { in at least } \\
3 \text { patients }\end{array}$ \\
\hline \multirow[t]{3}{*}{ KEGG } & ECM-receptor Interaction & 1 & $3.35 \times 10^{-11}$ & $\sim 4.5 X$ & $74 \%(62 / 84)$ & $40 \%(127 / 316)$ & 30 \\
\hline & Focal Adhesion & 2 & $2.62 \times 10^{-9}$ & $\sim 2.9 \mathrm{X}$ & (61\%) 122/199 & $58 \%(183 / 316)$ & 45 \\
\hline & Calcium Signaling & 3 & $2.05 \times 10^{-8}$ & $\sim 2.9 X$ & $63 \%(112 / 179)$ & $48 \%(153 / 316)$ & 40 \\
\hline \multirow[t]{3}{*}{ Gene Ontology } & Cell Adhesion & 1 & $1.03 \times 10^{-25}$ & $\sim 2.5 X$ & $64 \%(366 / 576)$ & $89 \%(281 / 316)$ & 156 \\
\hline & Developmental process & 14 & $1.03 \times 10^{-15}$ & $\sim 1.5 X$ & 49\% (430/869) & $90 \%(284 / 316)$ & 375 \\
\hline & Extracellular Matrix & 19 & $2.56 \times 10^{-14}$ & $\sim 2.8 X$ & $53 \%(80 / 150)$ & $40 \%(127 / 316)$ & 76 \\
\hline
\end{tabular}


pathways other than standard cancer pathways. Taken together, our data suggests that the spectrum of mutations in cell adhesion related genes is broad across this molecular function, with $64 \%$ of cell adhesion genes mutated at least once, and most interestingly with $89 \%$ of tumors observed to have at least one mutation.

The observations presented here are consistent with several reported studies on cell adhesion and tumorigenesis. Cell adhesion and extracellular matrix are known to be critical pathways in the metastatic process [15-17] and our results suggest there may be multiple genes, within the same pathway that EOC uses to achieve tumorogenic capability. Targeting of cell adhesion related genes agrees well with multiple previous reports of EOC specific gene expression changes in this pathway $[18,19]$. However, the breadth and prevalence of the mutational targeting of this pathway is a novel finding. Moreover, our results confirm recent findings that the ECM receptor interaction pathway is affected in ovarian cancer specifically and in many other cancers in general. In a recent study of cancer genetic deregulation at the transcriptomic level, the cell adhesion pathway was the most conserved for deregulation, showing enrichment in 26 tumor types [20]. In the same study, the ECM-receptor interaction pathway was found highly deregulated in many cancers including ovarian cancer.

The deregulation in the cell-adhesion and ECM pathway has broad implications. Indeed a study of EOC gene expression revealed agreement on an increase in ECM gene expression in chemotherapy resistant tumors [21]. Moreover, molecular subtypes of tumors, based on gene expression studies, have been identified and the categories encompassing high-grade serous EOC regularly include upregulated expression of ECM and cell adhesion genes [22]. A recent study of CNV in EOC primary tumors revealed an increase of extracellular matrix and cell adhesion genes in tumors with no CCNE1 amplification, yet with a short time to relapse [23]. Gene expression analysis of cisplatin resistant cancer cells revealed extracellular matrix related genes as a primary differentiator of chemotherapy resistance and Collagen 6A3 (COL6A3) has been shown to contribute to cisplatin resistance [24]. Our analysis agrees well with previous findings by Capo-chichi and colleagues that collagen IV and laminin are aberrantly expressed in primary ovarian tumors [25].

The importance of extracellular matrix and cell adhesion genes in EOC and other cancers is clear [26,27]. Other groups' gene expression studies, and our own analysis of both the prevalence and broad spectrum of mutation within cell adhesion gene groups reinforce the likely central role cell adhesion plays in epithelial ovarian cancers.

\section{Competing interests}

The authors declare that they have no competing interests.

\section{Authors' contributions}

AR envisioned, analyzed data and helped write the manuscript. $\mathrm{NMH}$ analyzed data and helped write the paper. JAM envisioned the study, analyzed data and wrote the manuscript. All authors read and approved the final manuscript.

\section{Author details}

'Stem cell and microenvironment laboratory, Weill Cornell Medical College in Qatar, Education city, Qatar Foundation, Doha, Qatar. ${ }^{2}$ Department of Genetic Medicine, Weill Cornell Medical College, NY, NY, USA. ${ }^{3}$ Genomics Core, Weill Cornell Medical College in Qatar, Education city, Qatar Foundation, Doha, Qatar. ${ }^{4}$ Department of Genetic Medicine, Weill Cornell Medical College, Genomics Core Laboratory, Weill Cornell Medical College in Qatar, Qatar-Foundation, Doha, Qatar.

Received: 26 July 2012 Accepted: 20 September 2012

Published: 24 September 2012

\section{References}

1. Jemal A, Siegel R, Ward E, et al: Cancer Statistics, 2006. CA Cancer J Clin 2006, 56:106-130.

2. Cannistra SA: Cancer of the ovary. N Engl J Med 2004, 351:2519-2529.

3. Chang S-J, Bristow RE: Evolution of surgical treatment paradigms for advanced-stage ovarian cancer: redefining "optimal" residual disease. Gynecol Oncol 2012, 125:483-492.

4. Chon HS, Lancaster JM: Microarray-based gene expression studies in ovarian cancer. Cancer Control 2011, 18:8-15.

5. Chien J, Fan JB, Bell DA, et al: Gynecologic Oncology Analysis of gene expression in stage I serous tumors identifies critical pathways altered in ovarian cancer. Gynecol Oncol 2009, 114:3-11.

6. Gómez-Raposo C, Mendiola M, Barriuso J, Hardisson D: Redondo a: Molecular characterization of ovarian cancer by gene-expression profiling. Gynecol Oncol 2010, 118:88-92.

7. Spentzos D, Levine DA, Kolia S, et al: Unique gene expression profile based on pathologic response in epithelial ovarian cancer. J Clin Oncol 2005, 23:7911-7918.

8. Berchuck A, Iversen ES, Lancaster JM, et al: Prediction of optimal versus suboptimal cytoreduction of advanced-stage serous ovarian cancer with the use of microarrays. Am J Obstet Gynecol 2004, 190:910-925.

9. Hartmann LC, Lu KH, Linette GP, et al: Gene expression profiles predict early relapse in ovarian cancer after platinum-paclitaxel chemotherapy. Clin Cancer Res 2005, 11:2149-2155.

10. Malek JA, Mery E, Mahmoud YA: Copy number variation analysis of matched ovarian primary tumors and peritoneal metastasis. PLOS One 2011, 6:e28561.

11. Malek JA, Martinez A, Mery E, et al: Gene expression analysis of matched ovarian primary tumors and peritoneal metastasis. J Trans/ Med 2012, 10:121.

12. Bell $D$, Berchuck $A$, Birrer $M$, et al: Integrated genomic analyses of ovarian carcinoma. Nature 2011, 474:609-615.

13. Huang DW, Sherman BT, Lempicki RA: Systematic and integrative analysis of large gene lists using DAVID bioinformatics resources. Nat Protoc 2009, 4:44-57.

14. Backes C, Keller A, Kuentzer J, et al: GeneTrail-advanced gene set enrichment analysis. Nucleic Acids Res 2007, 35:W186-W192.

15. Hirohashi S, Kanai Y: Cell adhesion system and human cancer morphogenesis. Cancer Sci 2003, 94:575-581.

16. Zetter BR: Adhesion molecules in tumor metastasis. Semin Cancer Biol 1993, 4:219-229.

17. Stetler-Stevenson WG, Aznavoorian S, Liotta LA: Tumor cell interactions with the extracellular matrix during invasion and metastasis. Annu Rev Cell Biol 1993, 9:541-573.

18. Heinzelmann-Schwarz VA, Gardiner-Garden M, Henshall SM, et al: Overexpression of the cell adhesion molecules DDR1, Claudin 3, and EpCAM in metaplastic ovarian epithelium and ovarian cancer. Clin Cancer Res 2004, 10:4427-4436. 
19. Gardner MJ, Jones LM, Catterall JB, Turner GA: Expression of cell adhesion molecules on ovarian tumour cell lines and mesothelial cells, in relation to ovarian cancer metastasis. Cancer Lett 1995, 91:229-234.

20. Krupp M, Maass T, Marquardt JU, et al: The functional cancer map: a systems-level synopsis of genetic deregulation in cancer. BMC Med Genomics 2011, 4:53.

21. Helleman J, Jansen MPHM, Span PN, et al: Molecular profiling of platinum resistant ovarian cancer. Int J Cancer 2006, 118:1963-1971.

22. Tothill RW, Tinker AV, George J, et al: Novel molecular subtypes of serous and endometrioid ovarian cancer linked to clinical outcome. Clin Cancer Res 2008, 14:5198-208.

23. Etemadmoghadam D, deFazio A, Beroukhim R, et al: Integrated genomewide DNA copy number and expression analysis identifies distinct mechanisms of primary chemoresistance in ovarian carcinomas. Clin Cancer Res 2009, 15:1417-1427.

24. Sherman-Baust CA, Weeraratna AT, Rangel LBA, et al: Remodeling of the extracellular matrix through overexpression of collagen $\mathrm{VI}$ contributes to cisplatin resistance in ovarian cancer cells. Cancer Cell 2003, 3:377-386.

25. Capo-Chichi CD, Smith ER, Yang D-H, et al: Dynamic alterations of the extracellular environment of ovarian surface epithelial cells in premalignant transformation, tumorigenicity, and metastasis. Cancer 2002, 95:1802-1815.

26. Cabodi S, delPilar Camacho-Leal M, Di Stefano P, Defilippi P: Integrin signalling adaptors: not only figurants in the cancer story. Nat Rev Cancer 2010, 10:858-870.

27. Desgrosellier JS, Cheresh DA: Integrins in cancer: biological implications and therapeutic opportunities. Nat Rev Cancer 2010, 10:9-22.

doi:10.1186/2043-9113-2-15

Cite this article as: Rafii et al: High-prevalence and broad spectrum of Cell Adhesion and Extracellular Matrix gene pathway mutations in epithelial ovarian cancer. Journal of Clinical Bioinformatics 2012 2:15.

\section{Submit your next manuscript to BioMed Central and take full advantage of:}

- Convenient online submission

- Thorough peer review

- No space constraints or color figure charges

- Immediate publication on acceptance

- Inclusion in PubMed, CAS, Scopus and Google Scholar

- Research which is freely available for redistribution 